\title{
HOW DOES HYBRIDIZATION INFLUENCE THE DECISION MAKING PROCESS IN CONSERVATION? THE GENUS ORCHIS (ORCHIDACEAE) AS A CASE HISTORY
}

\author{
Michael F. FAY ${ }^{1,3}$, R. J. SMith ${ }^{1}$, K. ZUIDERduin ${ }^{1}$, E. HoOPER ${ }^{1}$, R. SAMUEL ${ }^{2}$, \\ R. M. BATEMAN ${ }^{1} \&$ M. W. CHASE ${ }^{1}$ \\ ${ }^{1}$ Jodrell Laboratory, Royal Botanic Gardens, Kew, Richmond, Surrey, TW9 3DS, U.K. \\ ${ }^{2}$ Department of Systematic and Evolutionary Botany, Rennweg 14, 1030 Vienna, Austria \\ ${ }^{3}$ Author for correspondence: m.fay@kew.org
}

KEY wORDS: AFLP, hybridization, introgression, ITS rDNA, Orchis, plastid microsatellites

Hybridization is a fundamental process in biology and can lead to new evolutionary lineages. However, if the parental taxa involved are rare, difficult decisions may have to be made regarding the conservation of the biological process versus the conservation of the parental taxa. The genus Orchis in Europe is a good example of a group of species in which these types of questions arise as several of the species hybridize where they co-occur. The example used here relates to $O$. militaris, O. purpurea and $O$. simia in the anthropomorphic group (so called because the labellum has lobes thought to resemble arms and legs). All three species are widespread in Europe, although they are rare in large parts of their ranges, and they have substantial areas of overlap in distribution. All three are rare in Britain, occurring predominantly in south east England. Orchis militaris and O. simia and are only known from two and three natural sites in England, respectively. Orchis purpurea is less rare, but is still geographically localized.

Morphological inspection of plants provides convincing evidence of widespread hybridization in different parts of the range of these species. In addition, preliminary genetic data indicate that there is some introgression of genetic markers in populations that appear on a morphological basis to be representatives of one species rather than hybrids (Fay \& Krauss, 2003). Finally, we know of a number of populations in which hybridization is currently occurring, including one in England where $O$. purpurea has recently appeared at one $O$. simia site. The first hybrids flowered in 2006.
To investigate patterns of hybridization and introgression in these species further, we compiled data sets for three types of markers: sequences of the nuclear ribosomal spacer regions (nrITS), plastid microsatellites, and amplified fragment length polymorphisms (AFLP). These markers have now been applied to samples from populations of all three species and some putative hybrids, and we are currently expanding our sample size to include individuals from a wider geographical range.

The new ITS sequences were added to a subset of those in the matrix of Pridgeon et al. (1997), and phylogenetic analyses were carried out. The plastid microsatellite markers were developed for use with a range of Orchis spp., including the anthropomorphic group and their relative $O$. mascula. Five regions showing length variation within and between species were identified (as described in Fay and Krauss, 2003), and primers were designed to allow the amplification of short fragments (90-230 base pairs) that contained the length-variable regions. Then differences between individuals were detected by assessing the length of the amplified fragments using an automated sequencer. Together the alleles for these five regions provide a haplotype for each individual and a minimum spanning tree was constructed from the haplotypes. AFLP were carried out using a modified version of the protocol of Vos et al. (1995). The resulting $0 / 1$ matrix was analysed using principal coordinates analysis and neighbor joining.

All three data types can provide information relating to hybridization. Plastid DNA provide informa- 
tion relating to the maternal parent of any hybrids. ITS rDNA is part of the nuclear genome and it is inherited biparentally, but hybrids soon begin a process of gene conversion and lose one of the two parental copies that they initially possessed. For fairly recently synthesized hybrids, both parental ITS alleles are present, but for older hybrids only one of these alleles (often that from the maternal parent) normally remains. AFLP have been widely used in studies of hybridization, because hybrids show additivity between the profiles found in the parental species and the hybrids consequently fall in an intermediate position between the parents in ordination plots (e.g., Fay et al., 2003).

Four different ITS sequence types (each with minor variants) were identified, one each for $O$. militaris and $O$. simia and two for $O$. purpurea. In addition, some individuals of $O$. purpurea and $O$. simia had the ITS copy type of $O$. militaris. Individuals of intermediate morphology (presumed recent hybrids) had ITS copies from both putative parents. Plastid microsatellites did not provide such a clear division, but there were two major clusters identified with these markers, consisting predominantly of $O$. militaris and $O$. simia, respectively, but each also containing some individuals of $O$. purpurea. Most hybrids and some individuals of $O$. simia fell in the $O$. militaris cluster. AFLP identified distinct clusters for the three species, and the cluster for $O$. purpurea was divided into two subclusters. Hybrids fell in intermediate positions with AFLP as expected.

These data indicate that $O$. militaris and $O$. simia are good species that sometimes hybridize, leading to occasional plastid and ITS capture. In all individuals showing evidence of introgression and hybrids studied, plastid DNA indicated that $O$. militaris was the female parent. While our data set is still somewhat limited, it thus appears that hybridization occurs predominantly in one direction in these species. The situation with $O$. purpurea was not, however, so clear. With ITS and with AFLP, there is a suggestion that this species as currently circumscribed includes two genetic entities. In contrast, we have still to identify a plastid type characteristic of this species, if one exists. Many English populations always believed to be $O$. purpurea contain individuals with the ITS type normally associated with $O$. militaris, and there is a possibility that this is the situation elsewhere in Europe. The situation is clearly complicated, and to resolve the status of $O$. purpurea, we will need to improve our sample size for both molecular and morphological studies.

How does all this relate to conservation? Hybridization between species in this group is clearly part of an ongoing process, and the species involved appear to be able to maintain pure populations despite this process. At the edge of the species range, however, there is a risk that the peripheral populations of pure individuals may be lost due to hybridization. In England, O. purpurea has expanded its range in recent years, possibly due the warmer summers, and it has now formed a population sympatric with one of the two remaining natural populations for $O$. simia. To the dismay of the managers of the nature reserve, several plants flowering for the first time in 2006 were morphologically intermediate and genetic evidence has shown them to be hybrids with $O$. purpurea as the female parent. As both species are rare in England, this situation has received widespread attention, and we are currently discussing the situation with the managers of the site and with the national conservation agency, Natural England. Various suggestions have been made, ranging from letting nature take its course to digging up the hybrids and the individuals of $O$. purpurea to protect the remaining pure individuals of $O$. simia. No decision has yet been made, but this should be done before the plants flower again in the 2007 season.

This and other examples of hybridization in European orchids will be used to illustrate the decision-making processes involved and the relative merits of conservation of named species versus the conservation of process.

\section{LiteratuRe Cited}

Fay, M.F., R.S. Cowan \& D.A. Simpson. 2003. Hybridisation between Schoenoplectus tabernaemontani and $S$. triqueter (Cyperaceae) species in the British Isles. Watsonia 24 : 433-442.

Fay, M.F. \& S.L. Krauss. 2003. Orchid conservation genetics in the molecular age. Pp. 91-112 in: K.W. 
Dixon, S.P. Kell, R.L. Barrett \& P.J. Cribb (eds.). relationships and polyphyly of Orchis sensu lata. Orchid conservation. Natural History Publications, Kota Kinabalu, Sabah. Lindleyana $12: 89-109$.

Pridgeon, A.M., R.M. Bateman, A.V. Cox, J.R. Vos, P., R. Hogers, M. Bleeker, M. Rijans, T. Van de Lee, M. Hornes, A. Frijters, J. Pot, M. Kuiper \& Hapeman \& M.W. Chase. 1997. Phylogenetics of subtribe Orchidinae (Orchidoideae, Orchidaceae) M. Zabeau. 1995. AFLP: a new technique for DNA based on nuclear ITS sequences. 1. Intergeneric fingerprinting. Nucleic Acids Research 23 : 44074414.

Mike Fay received his undergraduate degree in Genetics and Plant Breeding from the University College of Wales, Aberystwyth. His graduate studies were on Trifolium, and he received his Ph.D. from the University of Wales in 1989. He joined the staff of the Royal Botanic Gardens, Kew, in 1986 as Head of Micropropagation, giving him the opportunity to become involved professionally with orchids. In 1995, he established a conservation genetics program at Kew, and since 2002 he has been Head of Genetics. Many projects past and present are on orchids. He recently became Chair of the Orchid Specialist Group of IUCN. 